\title{
Multi-Objective Model Reference Adaptive Bus Priority Based on RFID
}

\author{
Guangyuan Wang \\ School of Computer Sci.\&Tech. \\ Dalian University of Technology \\ Dalian 116024, P.R.China \\ Email:dllgwgy@gmail.com
}

\author{
Guozhen Tan \\ School of Computer Sci.\&Tech. \\ Dalian University of Technology \\ Dalian 116024, P.R.China \\ Email:gztan@dlut.edu.cn
}

\author{
Yaodong Wang \\ School of Computer Sci.\&Tech. \\ Dalian University of Technology \\ Dalian 116024, P.R.China \\ Email:wyaodong@126.com
}

\begin{abstract}
A critical issue encountered in implementing a bus signal priority system is to ensure a tradeoff between the delay of a bus and the surrounding traffic. Traditional BSP methods treat buses uniformly so that an empty bus may have the same priority to the full loaded bus, and lead to an excessive delay for private traffic. However, with RFID technique, a control model can realize delicate control mode by identifying and acquiring more detailed, real-time and dynamic data on the bus. Hence, An intelligent method for determing bus priority based on RFID is proposed, assuming twoobjective optimization of both on-bus passengers' total delay and private vehicles total delay. The result is a considerable reduction in private traffic delay with higher traffic volume when using bus signal priority. What's more, the proposed control method could also be applied to the coordination of similar complex cyber-physical systems.
\end{abstract}

\section{INTRODUCTION}

In recent years, there is an increasing attention arising from Cyber Physical Systems(CPSs) that bring prospects to enable a modern grand vision for societallevel services that transcend space and time at scales never possible before. CPSs interconnect the cyber world with physical world via ubiquitously distributing heterogeneous sensors to the physical world. This shall bridge the gap between the cyber world, where information technology is built on computational discreteness, and physical world that is on the premise that the control principal is physical continuity[1]. This intimate coupling between the cyber and physical will be manifested from the nano-world to large-scale widearea systems at multiple time-scales. Applications of CPSs include, but not limited to, electric grid management, assisted living, transportation management, disaster recovery, industry automation, smart spaces, military applications, and environmental science research. Among many items mentioned above, three main prior areas have been identified and developed more fully: smart energy systems, intelligent transportation systems, and tele-healthcare systems[2].

CPSs make everything in the world interact with each other through omnipresent sensing nodes around environment. In this paper, we extend and integrate one of cutting-edge sensing nodes of CPSs which is named Radio-frequency identification (RFID) technique into the public traffic control systems. RFID technique has introduced or incorporated an easy use of the object (typically referred to as an RFID tag) into freights, vehicles, or even living things for the purpose of identifying and tracking the current-using radio waves.

Bus Signal Priority (BSP) is a prominent and effective urban transportation system which solves the question of how to carry more passengers, with limited traffic space to relieve traffic congestion. Over past 50 years, we have witnessed the evolution of BSP. Wilbur[3] studied transit signal priority manipulated manually. Bus actuated signal control was given by Vincent[4]. Chang considered the impact of bus preemption to other vehicles [5]. Liu proposed a kind of adaptive transit priority on corridors[6]. Along with the development of urban traffic control systems, much attention was devoted to the study of BSP integrated in these well-known systems such as UTCS/BPS[7], PRODYN[8], UTOPIA[9], SPPORT[10], SCOOT[11], OPAC[12] and RHODES[13].

\section{PROBLEM DESCRIPTION}

The aforementioned systems are designed to optimize some single performance index and multi performance indexes by weighted sum. In the optimal control of bus signal priority, it is considered that the bus should have the priority of crossing the intersection to reduce the delay. On the other hand, since buses share the same road resource with other traffic, it is self-evident that the bus priority strategy will lead to 
the negative effect, e.g., vehicles at non-bus phase suffering much longer queue. Thus how to offset the adverse performance of surrounding traffic while BSP control policy is well-implemented becomes a multiobjective optimization problem.

Because of the limitation of detecting means, traditional multi-objective BSP can only adopt rough granularity scheduling of which control strategy treats all buses uniformly, e.g., one bus, though with no passenger, probably obtains priority. About this problem, we introduce a new technique of RFID to realize delicate control mode.

Through the latest RFID sensing technique, control model can identify and access more detailed, realtime and dynamic data, which is beyond conventional technical capability, such as the classification between truck and bus, or the number of passengers on bus. The number of passengers on bus can be obtained by two infrared counters deployed on front door and rear door of bus. The front infrared counter can detect passengers on, while the rear one can detect passengers off. Then the difference value between them is stored into RFID in the bus and can be read into the reader. Through this means, the traffic control systems can get parameters of the number of passengers as well as bus position, etc, as exemplified in Fig.1.

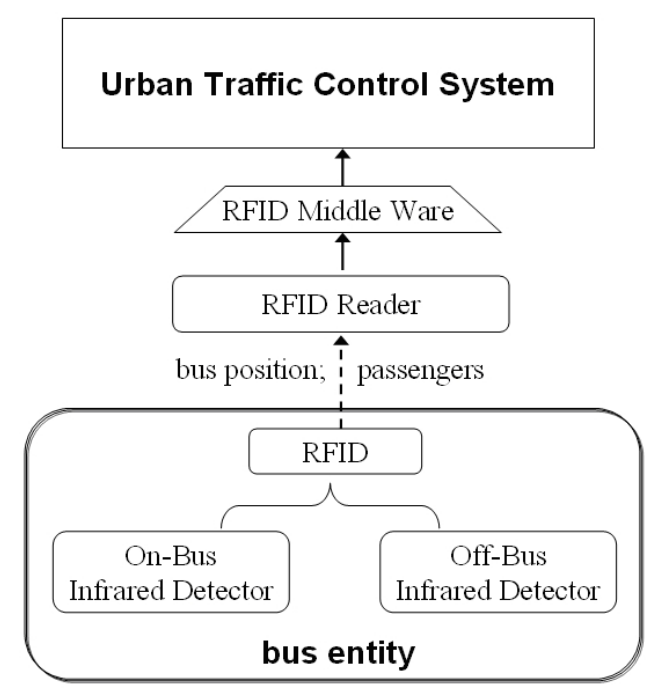

Fig. 1. RFID systems applied in bus entity

Due to the low cost and easiness of deployment, RFID systems display advantage compared with common conventional detectors. With RFID data on bus, traffic control system can capture abundant information to support finer-grained adaptive decision, e.g., in adap- tive signal system, by considering both the passengers' bus delay accessed from RFID and the non-bus traffic delay detected from loop vehicle detector, the control model can adjust signal plan support bus priority while decrease influence to private traffic at the same time.

We introduced the Multi-Objective Particle Swarm Optimization algorithm based on Crowd Distance (MOPSO-CD)[14] into our control model to settle the multi-objective optimization problem of bus priority. The two objectives are passengers' bus total delay and private vehicles total delay. Also, all traffic signals in this context are supposed to have merely two states: green and red, referring to go and wait respectively. No amber state is considered to simplify the problem.

\section{MODEL FORMULATION}

A primary objective for DMOSO model is to exhibit the signal control for adaptive BSP, through taking account of the BSP as well as the impact to surrounding traffic network.

In the following, we present a model for a novel adaptive bus signal priority based on RFID and multiobjective. Targeting at the multi-objective minimization problem, our proposed model with 2 decisive parameters and 2 objectives in this paper can be stated as:

$$
\begin{array}{r}
\min F(G)=\left[f_{1}(G), f_{2}(G)\right] \\
g_{i}^{l} \leq G_{i} \leq g_{i}^{u} \quad i=1,2
\end{array}
$$

Where the vector $G_{i}$ is effective green time for phase i. $g_{i}^{l}, g_{i}^{u}$ is respectively the lowerbound and upperbound of $G_{i} . f_{1}(G)$ denotes objective function for passengers' bus total delay, while $f_{2}(G)$ denotes objective function for non-bus vehicles total delay. As we have accurate number of passengers on bus, we can get balance between the bus and non-bus deliberately.

\section{A. Basic Model}

We formulate the problem with three basic models as:

(a) The final count of vehicles in queue:

$$
Q_{i}=Q_{i}^{\prime}+X_{i}-M_{i}\left(G_{i}\right)
$$

$Q_{i}$ denotes the final count of vehicles in queue of phase i. $Q_{i}^{\prime}$ denotes the initial count of vehicles in queue of phase i. $X_{i}$ denotes the count of vehicles arrived of phase $\mathrm{i}$ in the entire cycle T. $M_{i}\left(G_{i}\right)$ denotes the count of vehicles disappeared in $G_{i}$ time of phase i. 
(b) Vehicles delay model:

$$
\left\{\begin{aligned}
D_{i}^{k}= & \left(T-A_{i}^{k}+\frac{\sum_{c=X_{i}-Q_{i}+1}^{k-1} L_{i}^{c}}{V_{i}}\right) \\
& \text { if } k \geq X_{i}-Q_{i}+1 \\
D_{i}^{k}= & \max \left\{0, \sum_{1}^{i-1} G_{i}-A_{i}^{k}\right\} \\
& \text { otherwise }
\end{aligned}\right.
$$

The above equation $D_{i}^{k}$ provides a general expression for delay of the car, which is the kth vehicle arrived at phase $\mathrm{i}$ in the entire cycle T. $k$ represents the arrival index of vehicle at phase i. $A_{i}^{k}$ denotes the arrival time of kth vehicle to intersection at phase i. $L_{i}^{c}$ denotes the length of the cth car arrived to intersection at phase $\mathrm{i}$. $V_{i}$ denotes mean velocity of the car at phase i.

(c) bus weight model

The model proposed with bus priority is formulated based on providing traffic volume with a weight at relevant intersection. Thus a bus can be defined as a certain amount of ordinary cars by the weight. Considering passenger factor on bus, it implies the passengers' total delay to some intersection.

When detecting a bus towards the next intersection, information detecting system will capture running state of the bus and allocate some weight. This bus weighted function is up to the number of passengers on bus itself and the extent of bus delay:

$$
\begin{gathered}
W_{i}=n_{i}\left(1+f_{i}\right) \\
f_{i}= \begin{cases}0 & d_{i} \leq 0 \\
K d_{i} & d_{i} \geq 0\end{cases}
\end{gathered}
$$

Herein, $n_{i}$ represents the number of passengers on bus i. $d_{i}$ represents the delay extent where the value is negative if the bus arrival is earlier than schedule and positive if later.

Please notice two important features: First, The weight of $n$ passengers within one bus is equivalent to that of $\mathrm{n}$ private cars. In the no passenger state, the bus weight decreases to 0 . That means, the objective function just take into account the number of passengers. Second, the bus schedule is for reference to the delay extent of a bus, and the value is given by the difference between the detected bus arrival time and schedule. The larger the difference value is, the longer the delay is.

\section{B. Model of Objective}

(a) bus passengers' total delay

$$
P=\sum_{i=1}^{\phi} \sum_{k=1}^{x_{i}}\left(D_{i}^{k} W_{i}^{k}\right)
$$

$W_{i}^{k}$ denotes the weight of the bus which is the kth vehicle arrives to intersection at phase $i$. If the kth vehicle is not bus, $W_{i}^{k}$ is zero. $\phi$ denotes the phase number of the intersection.

(b) non-bus vehicles total delay

$$
C=\sum_{i=1}^{\phi} \sum_{k=1}^{x_{i}}\left(D_{i}^{k} b_{i}^{k}\right)
$$

$b_{i}^{k}$ is zero if the kth vehicle arrived at phase $\mathrm{i}$ is bus, otherwise 1 .

\section{SOLUTION ALGORITHM}

In this section, the BSP optimization is formulated as DMOSO model, of which solution algorithm is based on MOPSO. This algorithm extends the single-objective PSO to MOPSO domain. Via merging Crowd Distance into PSO algorithm, we can search the global optimum value and delete external set of nondominated solutions. MOPSO-CD contains mutated and constrained handling method, which addresses the problem of solution diversity and constrained optimization. We assume there are $\phi$ phases. So each particle has $\phi$ items. The ith item means the effective green time for the ith phase. The DMOSO algorithm for bus signal priority is given as following:

1) Initialize particle swarm: Set scale of swarm with $\mathrm{M}$, random particle $\mathrm{P}[\mathrm{i}]=\left\{G_{1}, G_{2}, \ldots, G_{\phi}\right\}$ and velocity $\mathrm{V}[\mathrm{i}]=\left\{v_{1}, v_{2}, \ldots, v_{\phi}\right\}=\{0,0, \ldots, 0\}$ for each particle.

2) Compute objective vector corresponding to each particle.

3) Initialize best value of each particle: PBESTS[i]=P[i], and that of swarm: GBEST $=\mathrm{P}[\mathrm{i}]$

4) Initialize iteration counter: $T=0$.

5) Store non-dominated solution vectors to extern set A.

6) Loop until iterator $=M$.

- a) Compute crowd distance. Order extern set.

- b) $\mathrm{V}[\mathrm{i}]=\mathrm{W} * \mathrm{~V}[\mathrm{i}]+\mathrm{R} 1 *(\operatorname{PBESTS}[\mathrm{i}]-\mathrm{P}[\mathrm{i}])+$ $\mathrm{R} 2 *(\mathrm{~A}[\mathrm{GBEST}]-\mathrm{P}[\mathrm{i}])$.

- c) $\mathrm{P}[\mathrm{i}]=\mathrm{P}[\mathrm{i}]+\mathrm{v}[\mathrm{i}]$. 
- d) Check P[i], V[i]. If P[i] goes beyond the boundary of maxmin green time, -v[i];

- e) Mutation P[i];

- f) Compute total delay functions for both bus passengers and private traffic, then update for each particle.

- g) Save non-dominated solutions.

7) Iterator $=$ M. End.

\section{SIMULATION RESULTS}

In this section, our experiment relevant to total delay of both bus passengers and private traffic will be discussed, with the aim of proving the efficiency of the proposed DMOSO control model. Due to the nature of this research, modeling and simulating methods are essential elements in the design and evaluation of experiments. In our simulation experiment, we have used the VISSIM traffic simulator, which supports microscopic traffic simulation exactly. In our performance study, the simulation environment is built to one fourleg intersection for BSP, as Fig.2 shows.

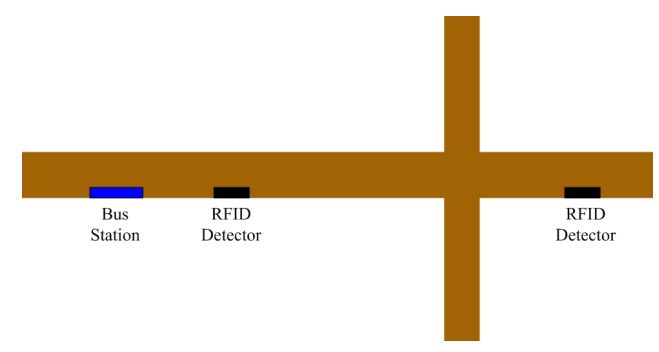

Fig. 2. Simulation Environment for BSP

The bus velocity is set to $18 \mathrm{~m} / \mathrm{h}$; bus passengers follow uniform distribution $(0,30)$; bus delay follow uniform distribution $(-30 \mathrm{~s},+30 \mathrm{~s})$; saturation flow is defined as 1800 vehicles per hour. Fix-time with no bus priority, actuated phase-extension bus priority[4] and DMOSO bus priority are simulated respectively and compared together. In MOPSO-CD algorithm, inertia weight uses 0.4 , initial particles of 100 , maximum number of iterations of 500, and a mutation rate of 0.5 . The data result of Private Traffic Total Delay (PTTD) and Bus Passengers Total Delay (BPTD) is shown in Table1.

Fig. 3 indicates that, with the increasing proportion of private vehicles, traditional actuated green-time extension can keep bus passengers' delay at a low level, however, the private traffic total delay rises up sharply; by using DMOSO model, it takes both bus and private traffic delays into consideration. As proportion
TABLE I

EXPERIMENT RESUlts: PTTD AND BPTD PERFORMANCE, ON FIX-TIME OPERATION BASELINE

\begin{tabular}{|c|c|c|c|}
\hline Private traffic & Mode & Reduce PTTD & Reduce BPTD \\
\hline \multirow{2}{*}{150} & Actuated BSP & $-0.51 \%$ & $58.33 \%$ \\
\cline { 2 - 4 } & DMOSO BSP & $3.43 \%$ & $62.50 \%$ \\
\hline \multirow{2}{*}{200} & Actuated BSP & $-4.27 \%$ & $80.54 \%$ \\
\cline { 2 - 4 } & DMOSO BSP & $-13.68 \%$ & $52.00 \%$ \\
\hline \multirow{2}{*}{300} & Actuated BSP & $6.76 \%$ & $72.22 \%$ \\
\cline { 2 - 4 } & DMOSO BSP & $16.22 \%$ & $47.22 \%$ \\
\hline \multirow{3}{*}{350} & Actuated BSP & $-5.03 \%$ & $68.82 \%$ \\
\cline { 2 - 4 } & DMOSO BSP & $8.47 \%$ & $39.78 \%$ \\
\hline \multirow{2}{*}{400} & Actuated BSP & $-37.26 \%$ & $64.17 \%$ \\
\cline { 2 - 4 } & DMOSO BSP & $-5.19 \%$ & $40.00 \%$ \\
\hline \multirow{2}{*}{450} & Actuated BSP & $-104.56 \%$ & $74.40 \%$ \\
\cline { 2 - 4 } & DMOSO BSP & $3.32 \%$ & $48.00 \%$ \\
\hline \multirow{2}{*}{500} & Actuated BSP & $-105.59 \%$ & $90.53 \%$ \\
\cline { 2 - 4 } & DMOSO BSP & $-4.90 \%$ & $61.15 \%$ \\
\hline & Actuated BSP & $-154.30 \%$ & $89.97 \%$ \\
\cline { 2 - 4 } & DMOSO BSP & $-3.64 \%$ & $40.88 \%$ \\
\hline
\end{tabular}

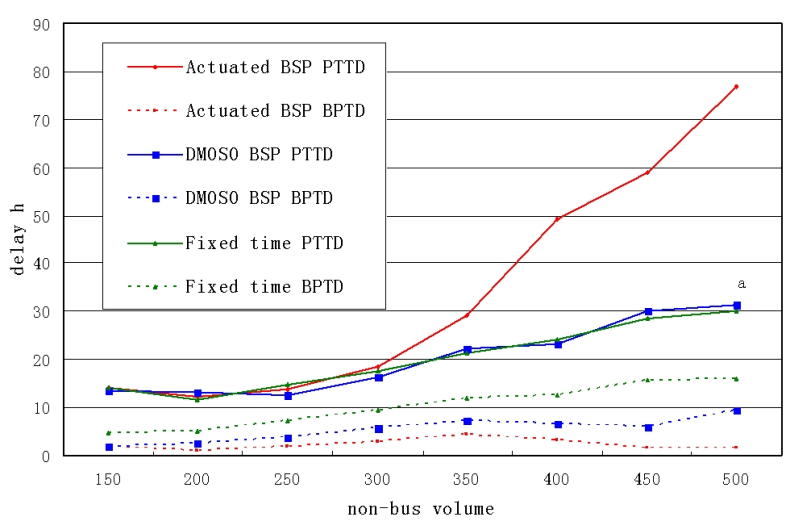

Fig. 3. Performance of fix-time without BSP, actuated phaseextension BSP and DMOSO BSP.

of private traffic rises up, bus priority weight gradually decreases, and private traffic delay changes little compared with fix-time operation. Moreover, if private traffic volume exceeds 350 in this case, there would be a significant benefit to hold back private traffic from degrading while DMOSO model is operated.

\section{CONCLUSION}

As a new era of CPSs is coming, a revolutionary transformation is inevitable for every aspect of traditional science and technology, including transportation control systems. In this paper, at first we have discussed 
the issue of BSP because of adverse effect to surrounding traffic, and some limitations in conventional priority control approaches at first. Then, we have proposed a new model for bus signal priority control based on promising RFID technique. The significance of this control model is that, with regard to a complex giant system such as transportation system, it allows us to detect further dynamic information as well as use intelligent methods to decrease both on-bus passengers' delay and non-bus vehicles delay, by which, we can carry out bus priority strategy and hold the surrounding traffic at non-bus phase back from degrading. Finally, as theoretical appeal of MOPSO, our proposed model is likely to be a promising approach to tackle the control problem of complicated traffic network, as CPSs style. Thus only the model be further extended, can it be applied to real wide-area traffic.

\section{REFERENCES}

[1] E. A. Lee, Cyber-Physical Systems - Are Computing Foundations Adequate. NSF Workshop On Cyber-Physical Systems: Research Motivation, Techniques and Roadmap, 2006.

[2] National Science Foundation. Report: Cyber-Physical Systems Summit[R]. St. Louis, Missouri. 2008, http://varma.ece.cmu.edu/Summit/CPS_Summit_ Report.pdf.

[3] W. Smith, W. Airbrake, Study of Evolutionary Urban Transportation. U.S. Department of Housing and Urban Development. Vol I-III. 1968.

[4] R. A. Vincent, B. R. Cooper, K. Wood, Bus actuated signal control at isolated intersections simulation studies of bus priority: report of Transport and Road Research Laboratory. Crowthorne: U.K., 1978.

[5] G. L. Chang, M. Vasudevan, C. Su, Bus preemption under adaptive signal control environment. Transportation Research Record 1494, 146-154, 1995.

[6] H. Liu, Skabardonis, A. Zhang, A dynamic model for adaptive bus signal priority. 82nd Transportation Research Board Annual Meeting, Washington DC, 2003.

[7] C. J. Macgowan, I. J. Fullerton, Development and testing of advanced control strategies in the urban traffic control system. Public Roads, 43(3):97-105, 1979.

[8] J. Henry, J. L. Farges, J. Tuffal, The PRODYN real time traffic algorithm. IFAC Symposium on Control in Transportation Systems, 305-310, 1983.

[9] V. Mauro, C. D. Taranto, UTOPIA. Proceedings of the 6th IFAC/IFIP/IFORS Symposium on Control, Computers, and Communication in Transportation, Paris, France, 245-252, 1989.

[10] M. Aonrad, F. Dion, S. Yagar, Real-time traffic signal optimization with transit priority: recent advances in the SPPORT model. 77th Annual Meeting of the Transportation Research Board, Washington DC, 1998.

[11] R.D. Bretherton, N.B. Hounsell, and B. Radia, Public Transport Priority in SCOOT. Proceedings of the 3rd Annual World Congress on ITS Systems, Orlando, FL, Oct. 14-18, 1996.

[12] N. H. Gartner, P. J. Tranoff, C. M. Anderews, Evaluation of the optimized policies for adaptive control (OPAC) strategy. Transportation Research Record, 1991.

[13] P. B. Mirchandani, A. Knyazyan, K. L. Head, An Approach Towards the Integration of Bus Priority, Traffic Adaptive Signal Control, and Bus Information/Scheduling Systems. Computer-Aided Scheduling of Public Transport, Springer Verlag, Germany, 319-334, 2001.

[14] C. R. Raquel, P.C. Naval, An effective use of crowding distance in multi-objective particle swarm optimization. Proceedings of the Genetic and Evolutionary Computation (GECCO2005), Washington DC, USA, 257-264, 2005. 